\title{
Simulation and Analysis of the Lunar Regolith Sampling Process Based on the Discrete Element Method
}

\author{
By Tianxi LiU, Cheng WeI, Lei LiAng, Jiang Zhang and Yang ZHAO \\ Department of Aerospace Engineering, School of Astronautics, Harbin Institute of Technology, Harbin, China
}

(Received November 27th, 2012)

\begin{abstract}
The regolith sampling process is risky but a key procedure in planet exploration. It not only accounts for the uncertainty of the soil particles that interacted with the bucket, but also the automated operation of the sampling manipulator. In this paper, the sampling process for lunar regolith, which involves the coupling between the microscopic lunar soil particles and macroscopic sampling mechanism, is analyzed. A discrete element model (DEM) that considers the torsion, bending and equivalent attraction between two particles is proposed for lunar soil modeling, whose microscopic parameters are calibrated based on tri-axial experimental results. Then a dynamic model for a lunar sampling manipulator is established using Lagrange formulations, and operation space control is applied to the manipulator to make the bucket move along the expected excavation trajectory. The numerical results show the whole sampling procedure, which considers the interaction between lunar soil and the bucket approaching a realistic situation. The operation space control schema is validated during the excavation simulation process, confirming the methods and model can support the performance analysis and design of a sampling mechanism for lunar explorations.
\end{abstract}

Key Words: Lunar Regolith Sampling, Discrete Element Method, Dynamic Simulation, Tool-soil Coupling Interaction, Operation Space Control

\section{Nomenclature}

$K_{n}$ : normal contact stiffness

$K_{t w}:$ torsional stiffness

$K_{s}:$ tangential contact stiffness

$K_{r}$ : bending stiffness

$\mu$ : friction coefficient

$\varepsilon_{n}$ : normal strain

$\boldsymbol{n}$ : normal unit vector

$\lambda_{d}$ : numerical damping coefficient

$C_{n}$ : critical normal cohesion

$K_{s}$ : tangential contact stiffness

$\boldsymbol{\varepsilon}_{s}:$ tangential strain

$\varphi$ : particle friction angle

$C_{s}$ : critical tangential cohesion

$d_{1}$ : distance between the contact point and particle $i$

$d_{2}$ : distance between the contact point and particle $j$

$\Theta_{t w}:$ relative torsional angle

$\Theta_{r}$ : relative bending angle

$\operatorname{Pr} j_{i}$ : component in a certain direction

$c$ : cohesion

$\phi$ : internal friction angle

$\sigma_{3}$ : confining pressure

$d$ : particle size

$E$ : particle elastic modulus

$v$ : tangential to normal stiffness ratio

$D$ : numerical damping coefficient

$\rho$ : particle density

$\boldsymbol{E}_{3}: 3 \times 3$ identity matrix

$\boldsymbol{H}_{b}$ : inertia matrix of the lunar rover space (base)
$\boldsymbol{H}_{c}$ : coupling inertial matrix between the base and manipulator

$\boldsymbol{H}_{m}$ : inertial matrix of manipulator

$\tilde{\boldsymbol{r}}$ : Cross-product matrix of vector $\boldsymbol{r}$

$\boldsymbol{k}_{i}$ : unit rotation vector of joint $i$

$\boldsymbol{r}_{g}$ : position vector of the sampling system centroid

$\boldsymbol{r}_{i}$ : centroid position vector of link $i$

$\boldsymbol{r}_{0}$ : centroid position vector of base body

$\boldsymbol{P}_{e}$ : position vector of the manipulator end effecter

$\boldsymbol{I}_{i}$ : inertia of link $i$ with respect to its centroid

$m_{i}$ : mass of link $i$

$\boldsymbol{c}_{b}$ : velocity-dependent nonlinear term for the base

$\boldsymbol{c}_{m}$ : velocity-dependent nonlinear term for the manipulator

$\boldsymbol{F}_{b}$ : external force and torque on the base

$\boldsymbol{\tau}_{m}$ : joint torque of the manipulator

$\boldsymbol{J}_{b}$ : Jacobi matrix from the end of the manipulator to the base

$\boldsymbol{J}_{m}$ : Jacobi matrix from the end of the manipulator to the joints

$\boldsymbol{F}_{h}$ : external force and torque on the end effecter

$\theta$ : joint rotation angles

$K_{p}$ : control parameters

$K_{d}$ : control parameters

$\boldsymbol{x}_{h d}:$ anticipated position

$\dot{\boldsymbol{x}}_{h d}$ : anticipated velocity

$\ddot{\boldsymbol{x}}_{h d}$ : acceleration of the manipulator end

$\boldsymbol{x}_{h}$ : actual position

$\dot{\boldsymbol{x}}_{h}$ : velocity of the manipulator end 


\section{Introduction}

In recent years, lunar exploration is regaining great attention throughout the world, and many countries that are ambitious in astronautics have begun researching lunar regolith sampling. Owing to the limited load capacity of rockets, it is necessary to optimize the performance of the sampling mechanism due to the external load. So it is very important to research the tool-soil coupling interaction in the lunar regolith sampling process. This research investigates the coupling between the lunar soil particles and the sampling mechanism under automated operation, and will be conducive to the optimal design for the sampling mechanism.

Generally, the sampling depth of lunar soil that is less than $1 \mathrm{~m}$ is called lunar regolith sampling. The usual sampling procedure is to excavate lunar regolith using a manipulator with a shovel-shaped end effecter carried by a lunar rover. ${ }^{1,2)}$ The contact forces between the bucket and discrete lunar soil particles is affected by the external load on the end of the manipulator. During the sampling process, the lunar soil deforms and is broken down by the sampling mechanism. Therefore, predicting the external load is extremely complex but very significant in designing the mechanism. In addition, the physical and mechanical properties of the lunar regolith are very different from the soil on Earth, ${ }^{3)}$ which contributes to great difficulty when analyzing toolsoil coupling interaction.

The discrete element method (DEM) $)^{4-6)}$ is commonly used for soil modeling: Jiang presented a two-dimensional contact model considering granular material incorporating rolling resistance ${ }^{7)}$ and Van der Waals force. ${ }^{8)}$ Matsushima et al. described a procedure used to characterize the threedimensional grain shape of lunar soil and conducted simulations of lunar soil using image-based DEM. ${ }^{9,10)}$ Taylor studied the wheel deposition ${ }^{11)}$ and driving performance of the lunar rover, ${ }^{12-14)}$ and discussed the interaction between the wheels and soil using DEM. But only a few studies have been done on lunar soil modeling: Hong studied the load status of the flat plate at the moment when applying force to the soil using the numerical limit analysis method on the background of lunar and Mars exploration. ${ }^{15)}$ Bui et al. simulated and experimented with the process of the flat plate promoting the soil horizontally using DEM, and discussed the effect of gravity during the lunar soil excavation process. ${ }^{16,17)}$ The above studies only carried out a static analysis or dynamic analysis in simple motion, so a realistic sampling process and coupling analysis with the dynamics of the sampling manipulator system are required.

In this paper, a lunar regolith model in three-dimensional DEM that includes the torsion, bending and equivalent attraction between two particles is proposed. Then the whole sampling process, in which a circular arc-shaped bucket is used as the end effector, of the manipulator in a lunar rover is simulated. The dynamic model of a sampling manipulator system that interacts with lunar soil is established, and operation space control is applied to make the bucket move along the expected trajectory during the sampling process.
The simulation results support the performance analysis and design for sampling mechanisms in future lunar explorations.

\section{Model of the Lunar Regolith}

\subsection{Particle contact model}

Interactions between particles include not only traditional normal and tangential forces, but also torsional and bending torque, and a three-dimensional DEM of colliding particles with torsional and bending torques are shown in Fig. 1.

In Fig. 1, $i$ and $j$ are two particles in contact with each other, $K_{n}$ is the normal contact stiffness and $K_{t w}$ is the torsional stiffness. As plane vectors, the tangential and bending strain can be taken as two components along the $x$ axis and $z$ axis, respectively. Note that $K_{s x}$ and $K_{s z}$ are the tangential contact stiffness, $K_{r x}$ and $K_{r z}$ are bending stiffness, and $\mu_{x}$ and $\mu_{z}$ are friction coefficients. For simplicity, usually we can get:

$$
\begin{aligned}
K_{s x} & =K_{s z}=K_{s}, \quad K_{r x}=K_{r z}=K_{r} \\
\mu_{x} & =\mu_{z}=\tan \varphi
\end{aligned}
$$

\subsection{Normal and tangential contact forces}

Most conventional calculations do not consider the cases in which two particles are embedded in each other. For two separated particles, the mutual gravitational forces between them are usually ignored by adding a non-tensional connector or splitter. However, between real lunar soil particles, there still exists strong attractive forces; for instance, electrostatic attraction, Van der Waals force and so forth. In this model, those complicated forces are comprehensively regarded as an equivalent attraction against simple spring tension. Set a maximum boundary value as the attraction failure zone. The normal contact force is described as:

$$
\begin{aligned}
& \boldsymbol{F}_{n}^{\prime}=\boldsymbol{n} \min \left(K_{n} \varepsilon_{n}, C_{n}\right) \\
& \boldsymbol{F}_{n}=\left(1-\lambda_{d} \operatorname{sgn}\left(\boldsymbol{F}_{n}^{\prime} \cdot \boldsymbol{v}_{i}\right)\right) \boldsymbol{F}_{n}^{\prime}
\end{aligned}
$$

where $\varepsilon_{n}$ is the normal strain, $\boldsymbol{n}$ is the normal unit vector, $\lambda_{d}$ is the numerical damping coefficient to represent the global damping effects by introducing force $\Delta \boldsymbol{F}_{d}=$ $-\lambda_{d} \cdot \operatorname{sgn}(\boldsymbol{F} \cdot \boldsymbol{v}) \boldsymbol{F}$, and $C_{n}$ is the critical normal cohesion coefficient to represent the maximum normal component

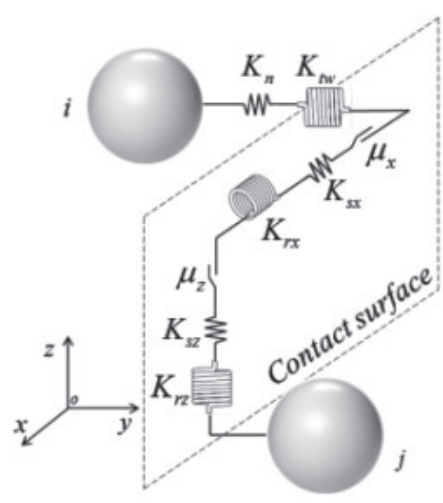

Fig. 1. Three-dimensional contact model of particles. 


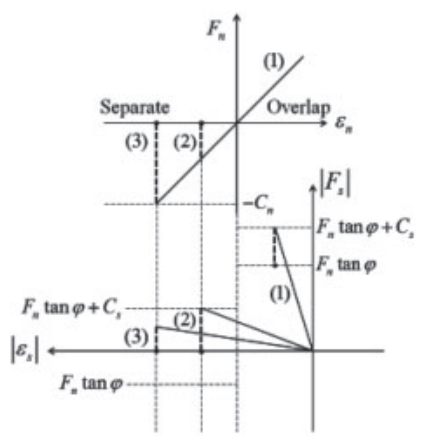

Fig. 2. Patterns of normal and shear contact forces.

of equivalent attraction.

Meanwhile, tangential contact force can be expressed as:

$$
\begin{aligned}
\boldsymbol{F}_{s}^{\prime} & =\frac{\boldsymbol{\varepsilon}_{s}}{\left|\boldsymbol{\varepsilon}_{s}\right|} \min \left(\left|K_{s} \boldsymbol{\varepsilon}_{s}\right|,\left|\boldsymbol{F}_{n}\right| \tan \varphi+C_{s}\right) \\
\boldsymbol{F}_{s} & =\left(1-\lambda_{d} \operatorname{sgn}\left(\boldsymbol{F}_{s}^{\prime} \cdot \boldsymbol{v}_{i}\right)\right) \boldsymbol{F}_{s}^{\prime}
\end{aligned}
$$

where $K_{s}$ is the tangential contact stiffness, $\boldsymbol{\varepsilon}_{s}$ is the tangential strain, $\varphi$ is the friction angle of particle, and $C_{S}$ is the critical tangential cohesion to represent the maximum tangential component of equivalent attraction.

When the normal or tangential contact force reaches the maximum, three cases appear according to different relative positions and their history paths (Fig. 2):

(1) When tangential contact force reaches its maximum first, the two particles are still embedded in each other and the tangential contact force changes from $F_{n} \tan \varphi+C_{s}$ to $F_{n} \tan \varphi$ due to the growth restriction of the tangential contact force.

(2) When tangential contact force reaches its maximum first, the two particles are already separated, so the tangential contact force changes from $F_{n} \tan \varphi+C_{s}$ to 0 . The normal contact force becomes 0 and the pattern of the particles enters the equivalent attraction failure zone.

(3) When normal contact force reaches its maximum first, the two particles are certainly separated since the normal contact force is limitless when embedded. The tangential and normal contact forces become 0 simultaneously, and the pattern of the particles enters the equivalent attraction failure zone.

In the direction of centroid connection vector between two particles, the normal contact force does not produce additional torque. Perpendicular to the centroid connection vector, the tangential contact force acts on the contact points and produces additional torque transferred to the centroid of the two particles:

$$
\begin{aligned}
& \boldsymbol{M}_{A 1}=d_{1}(-\boldsymbol{n}) \times \boldsymbol{F}_{s} \\
& \boldsymbol{M}_{A 2}=d_{2} \boldsymbol{n} \times \boldsymbol{F}_{s}
\end{aligned}
$$

where $d_{1}$ and $d_{2}$ are the distances between the contact points in particle $i$ and particle $j$.

\subsection{Torsional and bending torques}

Torsion and bending are the major torques between the particles, and have a huge effect on the shearing strength of the lunar soil. First, we establish the relationship between
Table 1. Cohesion and internal friction angle. ${ }^{18)}$

\begin{tabular}{cccccc}
\hline \multirow{2}{*}{ Depth } & \multicolumn{2}{c}{ Cohesion $c(\mathrm{kPa})$} & & \multicolumn{2}{c}{$\begin{array}{c}\text { Internal friction } \\
\text { angle } \phi\left(^{\circ}\right)\end{array}$} \\
\cline { 2 - 3 } \cline { 5 - 6 } \cline { 5 - 6 } & Range & Mean value & & Range & Mean value \\
\hline $0-15 \mathrm{~cm}$ & $0.44-0.62$ & 0.52 & & $41-43$ & 42 \\
$0-30 \mathrm{~cm}$ & $0.74-1.1$ & 0.9 & & $44-47$ & 46 \\
$30-60 \mathrm{~cm}$ & $2.4-3.8$ & 3.0 & & $52-55$ & 54 \\
$0-60 \mathrm{~cm}$ & $1.3-1.9$ & & & $48-51$ & \\
\hline
\end{tabular}

the relative angle and the difference of the angular velocity using iteration as follows:

$$
\left[\begin{array}{c}
\Theta_{t w} \\
\Theta_{r x} \\
\Theta_{r z}
\end{array}\right]=\left[\begin{array}{c}
\operatorname{Pr} j_{y} \\
\operatorname{Pr} j_{x} \\
\operatorname{Pr} j_{z}
\end{array}\right]\left(\omega_{1}-\omega_{2}\right) \mathrm{d} t
$$

where $\Theta_{t w}$ is the relative torsional angle, $\Theta_{r x}$ and $\Theta_{r z}$ are relative bending angles, and $\operatorname{Pr} j_{i}$ represents the component in a certain direction.

Then, the torsional and bending torques, in terms of defined contact stiffness, can be calculated as follows:

$$
\left[\begin{array}{c}
M_{t w} \\
M_{r x} \\
M_{r z}
\end{array}\right]=\left[\begin{array}{lll}
K_{t w} & & \\
& K_{r} & \\
& & K_{r}
\end{array}\right]\left[\begin{array}{c}
\Theta_{t w} \\
\Theta_{r x} \\
\Theta_{r z}
\end{array}\right]
$$

where $K_{t w}$ is torsional stiffness and $K_{r}$ is bending stiffness.

\subsection{Microscopic parameter calibration}

With the expectation of making the DEM model approach the real mechanical properties of lunar soil, it is necessary for the DEM model to take the tri-axial test to calibrate the microscopic parameter.

Macroscopic parameters of lunar soil show the mechanical properties of lunar soil, especially the cohesion, $c$, and the internal friction angle, $\phi$, which influence the shear strength. The commonly used data for cohesion and internal friction angle of lunar soil are shown in Table 1. ${ }^{18)}$

Microscopic parameters of the DEM model can be divided into three categories: (1) priori parameter, the confining pressure, $\sigma_{3}$ of tri-axial simulation; (2) fixed parameter, the particle size, $d$; and (3) variable parameters, particle friction angle, $\varphi$, particle elastic modulus, $E$, the tangential to normal stiffness ratio, $v$, the numerical damping coefficient, $D$, particle density, $\rho$, the critical normal cohesion, $C_{n}$, the critical tangential cohesion, $C_{s}$, the bending stiffness, $K_{r}$ and the torsional coefficient, $K_{t w}$.

We set up a tri-axial simulation test for calibration, taking $\sigma_{3}=50 \mathrm{kPa}$ in the contact analysis process of the singlevariable test. Considering the computational ability and efficiency, particle size $d$ cannot entirely take actual values. In this experiment, take the average particle radius as $1 \mathrm{~mm}$ and the linear distribution range as $0.5-1.5 \mathrm{~mm}$. By adjusting the variable parameters, the modified model equivalent to real lunar soil in macroscopic mechanical properties can be obtained.

Based on the tri-axial compression test for an actual lunar 


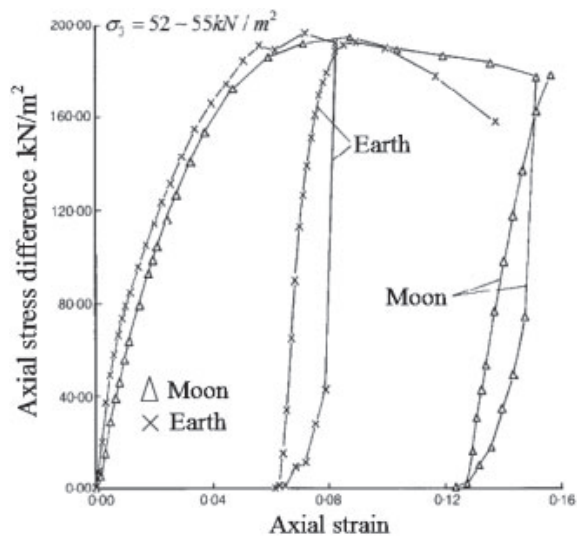

Fig. 3. Stress-strain curve of real lunar soil in a tri-axial test. ${ }^{19)}$

Table 2. Microscopic parameters of a calibrated DEM.

\begin{tabular}{lc}
\hline \multicolumn{1}{c}{ Parameters } & Values \\
\hline Particle friction angle $\left({ }^{\circ}\right)$ & 80 \\
Particle elastic modulus $(\mathrm{MPa})$ & 45 \\
Tangential to normal stiffness ratio & 0.5 \\
Numerical damping coefficient & 0.3 \\
Particle density $\left(\mathrm{kg} / \mathrm{m}^{3}\right)$ & 2900 \\
Critical normal cohesion $(\mathrm{kPa})$ & 0.9 \\
Critical tangential cohesion $(\mathrm{kPa})$ & 1.2 \\
Bending coefficient & 10 \\
Torsion coefficient & 3 \\
\hline
\end{tabular}

soil sample by $\operatorname{Scott}^{19)}$ (Fig. 3) under the confining pressure $53 \mathrm{kPa}$ on Earth, we can adjust five different sets of each microscopic parameter using the single-variable method until the stress-strain curves approach the results in Fig. 3.

Finally, the microscopic parameters of a DEM whose macroscopic mechanical properties coincide with real lunar regolith are calibrated in Table 2 .

Then, we can make use of calibrated microscopic parameters, and three stress-strain curves are obtained under simulations with three different confining pressures, as shown in Fig. 4.

Take the peak of the stress-strain curve as the major principal stress in each group, $\sigma_{1}=249.2 \mathrm{kPa}, 358.2 \mathrm{kPa}$, $490.3 \mathrm{kPa}$ and $\sigma_{3}=50 \mathrm{kPa}, 75 \mathrm{kPa}$, and $100 \mathrm{kPa}$, respectively. Mohr's circles are drawn based on the three sets of values. Even if the shear strength envelope is theoretically tangent to Mohr's circle, its accuracy cannot be assured due to the unavoidable errors in experiments. Results of the shear strength envelope, which can be obtained using the least-squares method, are shown in Fig. 5.

As a result, the cohesion, $c$, is $0.97 \mathrm{kPa}$ and the internal friction angle, $\phi$, is $42.03^{\circ}$, which can satisfy the requisite performance for simulating real lunar soil.

\section{Dynamics and Control of the Sampling Manipulator}

\subsection{Model assumption}

A lunar rover carries a sampling manipulator composed

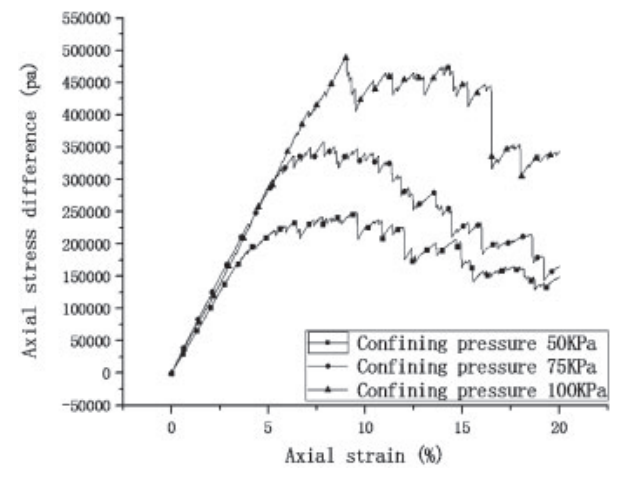

Fig. 4. Tri-axial test results for different confining pressures.

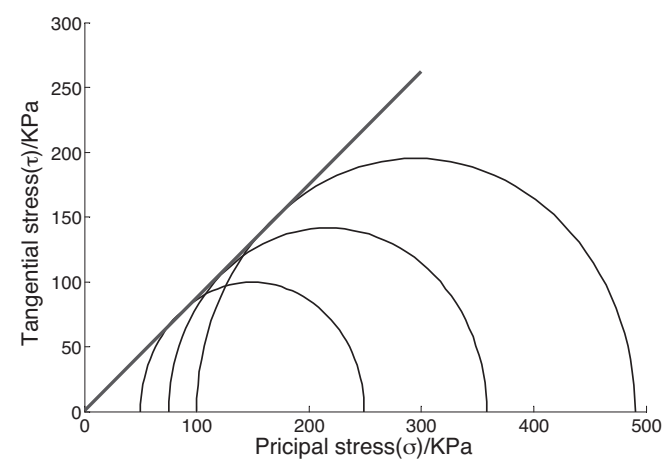

Fig. 5. Mohr's circle and shear strength envelope.

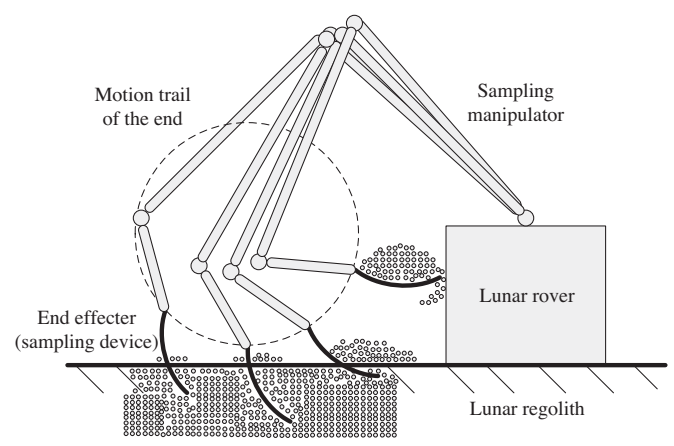

Fig. 6. Lunar regolith sampling illustration.

of three links and a bucket at the end of the final link. The manipulator has three rotational degrees of freedom. When beginning to sample lunar regolith, the tip of the final link moves along a circular trajectory as lunar regolith is collected into the bucket as shown in Fig. 6 .

Assumptions in the dynamic model of the lunar rover and the sampling manipulator are as follows:

(1) Each link of the sampling manipulator is a rigid body.

(2) In the sampling process, the position and attitude of the base are fixed.

(3) The nonlinear characters for joint motors, such as clearance and flexibility, are neglected.

(4) At each moment of the sampling process, the forces applied on the bucket from related lunar regolith particles are combined and applied to the endmost point of the manipulator's final link as an external load. 


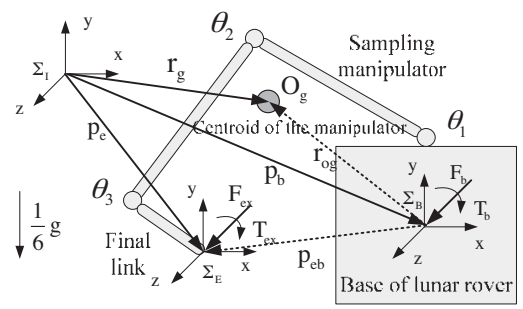

Fig. 7. Coordinate system of sampling manipulator and base model.

\subsection{Dynamics modeling}

According to the above assumptions, we define the coordinate system for the sampling manipulator and lunar rover as follows: the inertial coordinate system is $\Sigma_{I}$, the base coordinate system is $\Sigma_{B}$ with its origin at the centroid of the lunar rover, the end coordinate system is $\Sigma_{E}$. Joints are hinged around the $Z$-axis and the rotation angles, $\theta_{1}, \theta_{2}$ and $\theta_{3}$ (see Fig. 7).

A dynamic model for the lunar rover and sampling manipulator was established using Lagrange equations as follows:

$$
\begin{aligned}
& {\left[\begin{array}{ll}
\boldsymbol{H}_{b} & \boldsymbol{H}_{c} \\
\boldsymbol{H}_{c}^{\mathrm{T}} & \boldsymbol{H}_{m}
\end{array}\right]\left[\begin{array}{c}
\ddot{\boldsymbol{x}}_{b} \\
\ddot{\boldsymbol{\theta}}
\end{array}\right]+\left[\begin{array}{c}
\boldsymbol{c}_{b} \\
\boldsymbol{c}_{m}
\end{array}\right]=\left[\begin{array}{c}
\boldsymbol{F}_{b} \\
\boldsymbol{\tau}_{m}
\end{array}\right]+\left[\begin{array}{c}
\boldsymbol{J}_{b}^{\mathrm{T}} \\
\boldsymbol{J}_{m}^{\mathrm{T}}
\end{array}\right] \boldsymbol{F}_{h}} \\
& \boldsymbol{H}_{b}=\left[\begin{array}{cc}
w \boldsymbol{E}_{3} & w \tilde{\boldsymbol{r}}_{0 g}^{\mathrm{T}} \\
w \tilde{\boldsymbol{r}}_{0 g} & \boldsymbol{H}_{w}
\end{array}\right], \quad \boldsymbol{H}_{c}=\left[\begin{array}{c}
\boldsymbol{J}_{T g} \\
\boldsymbol{H}_{w \theta}
\end{array}\right] \\
& \boldsymbol{H}_{w}=\sum_{i=1}^{n}\left(I_{i}+m_{i} \tilde{\boldsymbol{r}}_{0 i}^{\mathrm{T}} \tilde{\boldsymbol{r}}_{0 i}\right)+\boldsymbol{I}_{0} \\
& w=\sum_{i=0}^{n}\left(m_{i}\right), \quad \boldsymbol{J}_{T g}=\sum_{i=1}^{n} m_{i} \boldsymbol{J}_{T i} \\
& \boldsymbol{H}_{w \theta}=\sum_{i=1}^{n}\left(\boldsymbol{I}_{i} \boldsymbol{J}_{R i}+m_{i} \tilde{\boldsymbol{r}}_{0 i} \boldsymbol{J}_{T i}\right) \\
& \boldsymbol{J}_{T i}=\left[\boldsymbol{k}_{1} \times\left(\boldsymbol{r}_{i}-\boldsymbol{p}_{1}\right), \cdots, \boldsymbol{k}_{i} \times\left(\boldsymbol{r}_{i}-\boldsymbol{p}_{i}\right), 0, \cdots, 0\right] \\
& \boldsymbol{H}_{m}=\sum_{i=1}^{n}\left(\boldsymbol{J}_{R i}^{\mathrm{T}} \boldsymbol{I}_{i} \boldsymbol{J}_{R i}+m_{i} \boldsymbol{J}_{T i}^{\mathrm{T}} \boldsymbol{J}_{T i}\right) \\
& \boldsymbol{J}_{R i}=\left[\boldsymbol{k}_{1}, \boldsymbol{k}_{2}, \cdots, \boldsymbol{k}_{i}, 0, \cdots, 0\right] \\
& \boldsymbol{J}_{b}=\left[\begin{array}{cc}
\boldsymbol{E}_{3}, & -\tilde{\boldsymbol{p}}_{0 e} \\
0, & \boldsymbol{E}_{3}
\end{array}\right], \quad \boldsymbol{p}_{0 e}=\boldsymbol{p}_{e}-\boldsymbol{r}_{0} \\
& \boldsymbol{J}_{m}=\left[\begin{array}{l}
\boldsymbol{k}_{1} \times\left(\boldsymbol{p}_{e}-\boldsymbol{p}_{1}\right), \cdots, \boldsymbol{k}_{n} \times\left(\boldsymbol{p}_{e}-\boldsymbol{p}_{n}\right) \\
\boldsymbol{k}_{1}, \boldsymbol{k}_{2}, \cdots, \boldsymbol{k}_{n}
\end{array}\right] \\
& \boldsymbol{r}_{0 g}=\boldsymbol{r}_{g}-\boldsymbol{r}_{0}, \quad \boldsymbol{r}_{0 i}=\boldsymbol{r}_{i}-\boldsymbol{r}_{0}
\end{aligned}
$$

\subsection{Operation space control}

In the sampling process, lunar regolith is broken down by the sampling device. Therefore, the contact force changes continuously and it is considered as the variable external load of the manipulator. We apply operation space control to the manipulator, so the manipulator can sample lunar regolith according to the expected tip trajectory with the variable external load from the lunar soil.
According to the bottom part of the dynamic equation (7):

$$
\boldsymbol{H}_{c}^{\mathrm{T}} \ddot{\boldsymbol{x}}_{b}+\boldsymbol{H}_{m} \ddot{\boldsymbol{\theta}}+\boldsymbol{c}_{m}=\boldsymbol{\tau}_{m}+\boldsymbol{J}_{m}^{\mathrm{T}} \boldsymbol{F}_{h}
$$

The base of the lunar rover effected by gravity and the lunar regolith is fixed on the surface of the moon, so $\ddot{\boldsymbol{x}}_{b}=\mathbf{0}$, and the control torque is simplified as:

$$
\boldsymbol{\tau}_{m}=\boldsymbol{H}_{m} \ddot{\boldsymbol{\theta}}+\boldsymbol{c}_{m}-\boldsymbol{J}_{m}^{\mathrm{T}} \boldsymbol{F}_{h}
$$

The control torque can be obtained by the operation space control schema:

$$
\begin{aligned}
\boldsymbol{\tau}_{m}= & \boldsymbol{H}_{m} \boldsymbol{J}_{m}^{\mathrm{T}}\left[\ddot{\boldsymbol{x}}_{h d}+K_{p}\left(\boldsymbol{x}_{h d}-\boldsymbol{x}_{h}\right)\right. \\
& \left.+K_{d}\left(\dot{\boldsymbol{x}}_{h d}-\dot{\boldsymbol{x}}_{h}\right)\right]+\boldsymbol{c}_{m}-\boldsymbol{J}_{m}^{\mathrm{T}} \boldsymbol{F}_{h}
\end{aligned}
$$

The velocity of the end tip is obtained by:

$$
\dot{\boldsymbol{x}}_{h}=\boldsymbol{J}_{m} \dot{\boldsymbol{\theta}}+\boldsymbol{J}_{b} \dot{\boldsymbol{x}}_{b}
$$

Defining $\boldsymbol{e}=\boldsymbol{x}_{h}-\boldsymbol{x}_{h d}$ as the operation space position error of the tip, and substituting it into the dynamic equation, the operation space error equation, which is an ordinary differential equation, can be obtained:

$$
\ddot{\boldsymbol{e}}+K_{d} \dot{\boldsymbol{e}}+K_{p} \boldsymbol{e}=0
$$

\section{Dynamic Simulation of the Sampling Process}

\subsection{Simulation condition}

We set a rectangular box as the container of the lunar particles. The box is $0.5 \mathrm{~m}$ in length, $0.1 \mathrm{~m}$ in height and $0.09 \mathrm{~m}$ in width. The shape of the bucket is a quarter of a circular arc with a radius of $0.1 \mathrm{~m}$. The width of the bucket is $0.06 \mathrm{~m}$. The bucket starts from left of the box and rotates around a certain point during the sampling process. The rotation angular velocity is $25^{\circ} / \mathrm{s}$. The particle radius is set as that described in Section 2.4. The micro-parameters of the lunar regolith are set as stipulated in Table 2 . The gravity on the moon is $1.633 \mathrm{~m} / \mathrm{s}^{2}$.

\subsection{Dynamic simulation of lunar soil}

The dynamic simulation process for lunar regolith sampling is shown in Fig. 8.

Figure 8 shows the sampling process. The bucket moves along an arc and lunar regolith is collected. Some of the particles in the bucket fall out due to the gravity. Sub-graphs (c) and (d) indicate that, after excavating, a trench appears and the particles on both sides of the trench do not fall back into the trench. This phenomenon is in agreement with the test results of the Surveyor 7 spacecraft on the lunar surface. ${ }^{20)}$

At any moment during the sampling process, the real-time positions, velocities and forces of all the lunar regolith particles are monitored. The final mass of the sample soil in the bucket is counted as $287.07 \mathrm{~g}$. Figure 9 shows the applied force and torque curves of the bucket during the sampling process. While excavation depth increases, more particles move into the bucket which generates resistance contact force on the bucket. Towards the end of the process, as the bucket begins to retract from the soil, the resistance decreases. The amount of particles in the bucket gradually sta- 

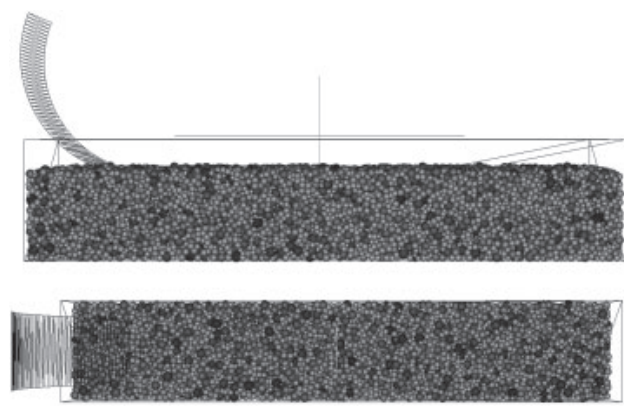

(a) Beginning stage

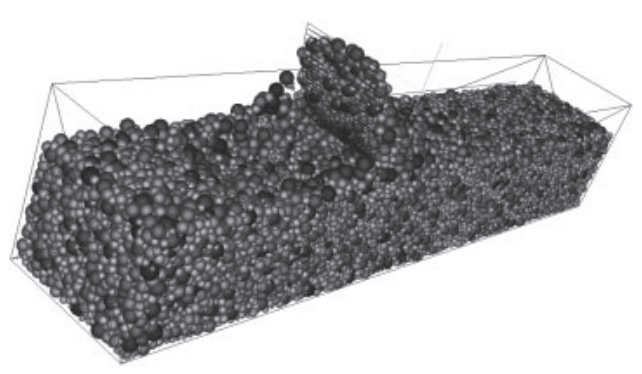

(b) Middle stage

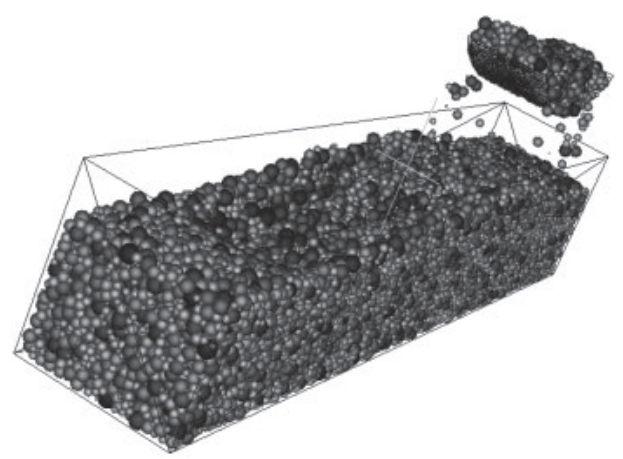

(c) Ending stage

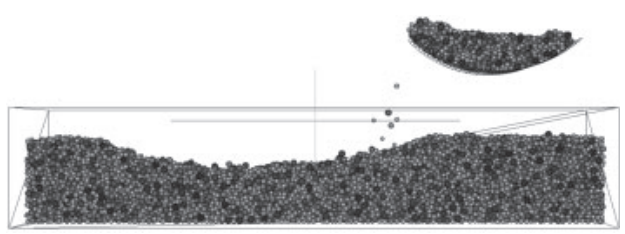

(d) Ending stage (sectional view)

Fig. 8. Lunar regolith sampling process

bilizes, and the vibrations caused by the force and torque on the bucket begin to dissipate. Finally, the force and torque applied to the bucket only account for the gravity of the lunar soil sample, which is very small as shown in Fig. 9. The resultant force is less than $25 \mathrm{~N}$, and the resultant torque is less than $5 \mathrm{Nm}$.

\section{Tool-soil Coupling Interaction Analysis}

\subsection{Simulation condition}

Dynamic properties of the lunar rover and sampling manipulator are shown in Table 3.

The tip of the manipulator moves around a fixed point

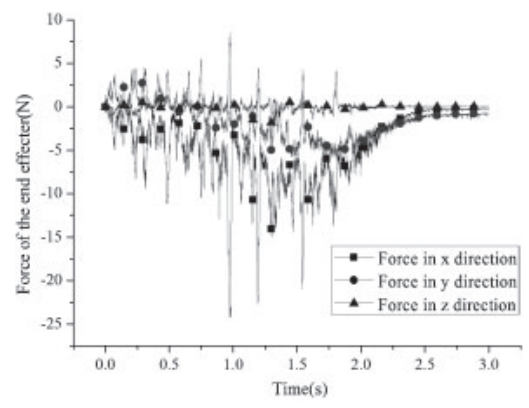

(a) Forces in different directions

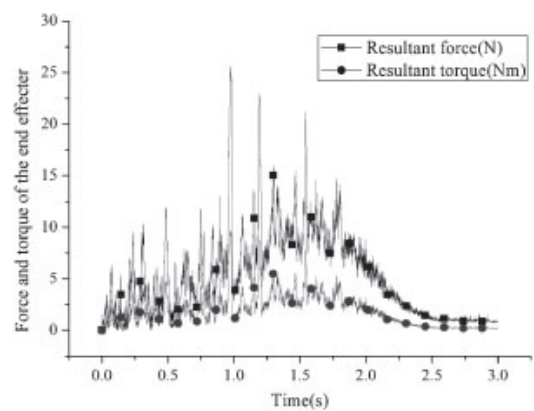

(b) Resultant force and torque

Fig. 9. Forces and torques on the tip of the manipulator.

Table 3. Physical parameters of the sampling manipulator.

\begin{tabular}{lrccc}
\hline Parameters & Lunar rover & Link 1 & Link 2 & $\begin{array}{c}\text { Link 3 } \\
\text { (without bucket) }\end{array}$ \\
\hline Mass $/ \mathrm{kg}$ & 1000 & 10 & 10 & 5 \\
Length $/ \mathrm{m}$ & 1 & 1 & 1 & 0.4 \\
$I_{x x} / \mathrm{kgm}^{2}$ & 100 & 1 & 1 & 1 \\
$I_{y y} / \mathrm{kgm}^{2}$ & 100 & 0.1 & 0.1 & 0.1 \\
$I_{z z} / \mathrm{kgm}^{2}$ & 100 & 1 & 1 & 1 \\
\hline
\end{tabular}

$I_{x x}, I_{y y}$, and $I_{z z}$ are the principal moments of inertia of each body.

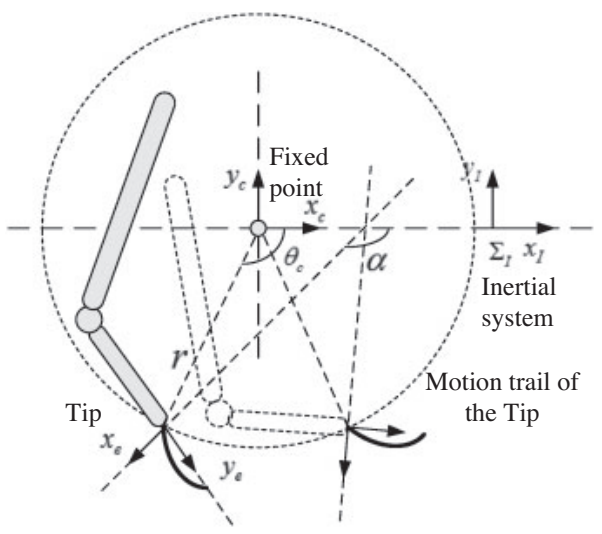

Fig. 10. Expected motion of bucket and tip of link.

$\left(x_{c}, y_{c}\right)$ and along a circle with the radius of $r$. The angle between the vector from the fixed point to the tip and the $+x$ direction of the inertial system is $\theta_{c}$, which changes as $\theta_{c}=\theta_{0}-\omega t$. The attitude angle of the bucket is $\alpha=\theta_{c}+45^{\circ}$. The relationship of these parameters is shown in Fig. 10. 
So the expected position, velocity and acceleration of the tip are obtained as follows:

$$
x_{h d}=\left[\begin{array}{c}
x_{c}+r \cos \left(\theta_{0}-\omega t\right) \\
y_{c}-r \sin \left(\theta_{0}-\omega t\right) \\
{\left[\begin{array}{lll}
0 & 0 & 0
\end{array}\right]^{\prime}} \\
\theta_{0}-\omega t+45^{\circ}
\end{array}\right]
$$

$$
\begin{aligned}
& \dot{x}_{h d}=\left[\begin{array}{c}
\omega r \sin \left(\theta_{0}-\omega t\right) \\
\omega r \cos \left(\theta_{0}-\omega t\right) \\
{\left[\begin{array}{ccc}
0 & 0 & 0
\end{array}\right]^{\prime}} \\
-\omega
\end{array}\right] \\
& \ddot{x}_{h d}=\left[\begin{array}{c}
-\omega^{2} r \cos \left(\theta_{0}-\omega t\right) \\
\omega^{2} r \sin \left(\theta_{0}-\omega t\right) \\
{\left[\begin{array}{llll}
0 & 0 & 0 & 0
\end{array}\right]^{\prime}}
\end{array}\right]
\end{aligned}
$$

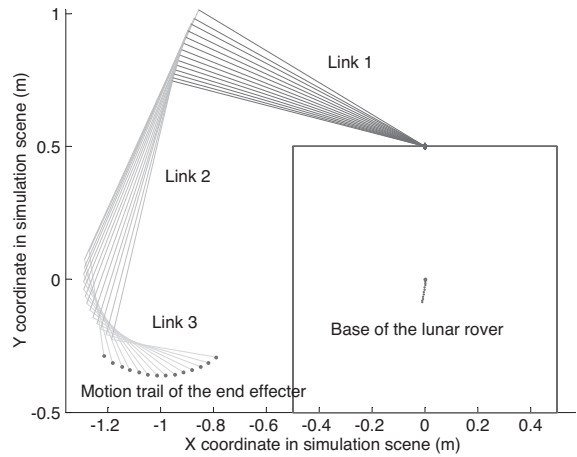

Fig. 11. Simulation process illustration.

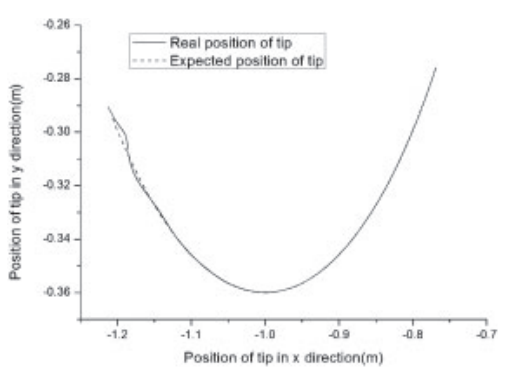

Fig. 12. Trajectory tracking of tip.

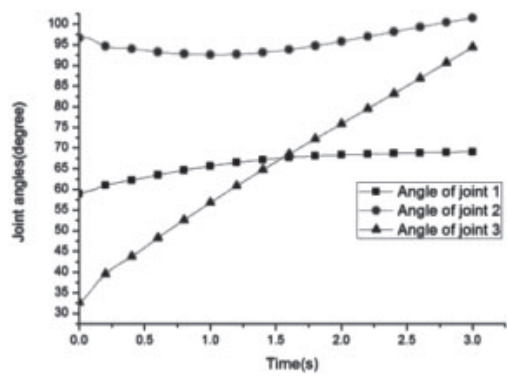

(a) Variation of joint angle

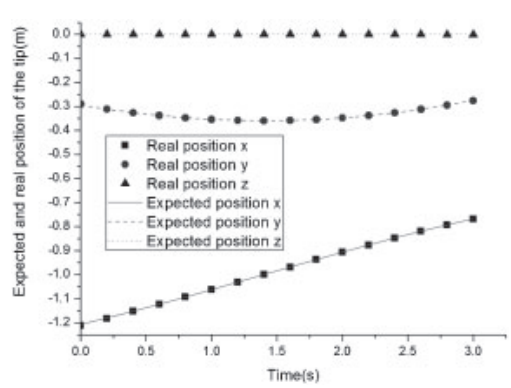

(b) Position of the tip

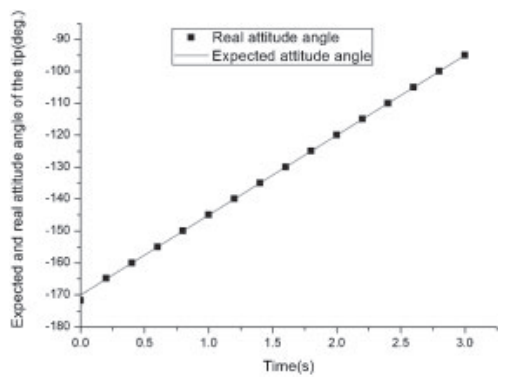

(c) Attitude angle of the tip

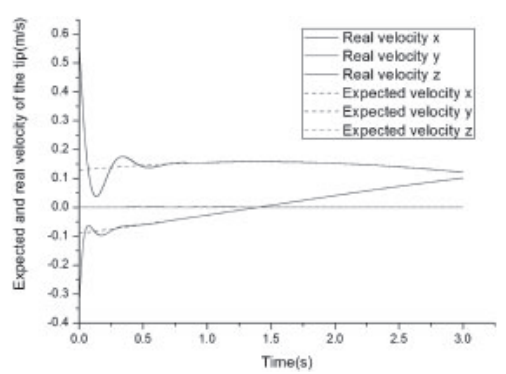

(d) Displacement velocity of the tip

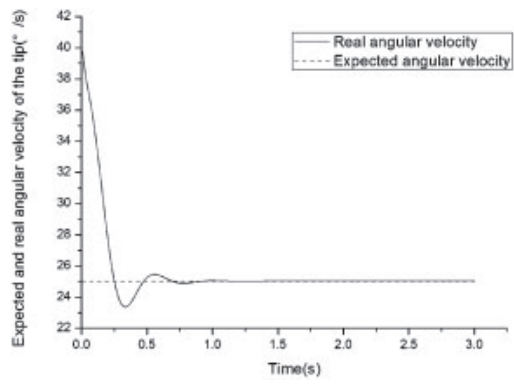

(e) Angular velocity of the tip

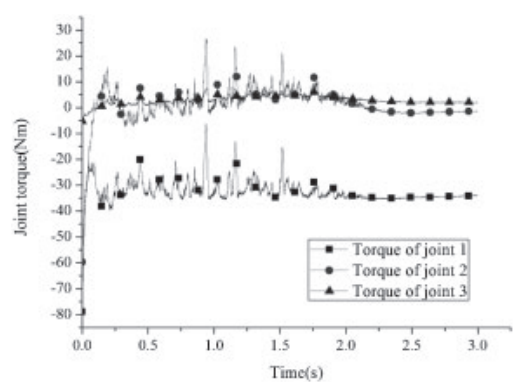

(f) Variation in joint torque

Fig. 13. Simulation results. 
When $x_{c}=-1 \mathrm{~m}, y_{c}=0 \mathrm{~m}, \theta_{0}=125^{\circ}, \omega=25^{\circ} / \mathrm{s}$, $r=0.36 \mathrm{~m}$ and $t \in[0,3] \mathrm{s}$, the initial angles of the three joints are $\theta_{1}=58.85^{\circ}, \theta_{2}=96.56^{\circ}$ and $\theta_{3}=32.59^{\circ}$, and the control parameters for the manipulator are $K_{p}=1000$ and $K_{d}=50$.

\subsection{Simulation and analysis for tool-soil coupling}

The manipulator moves along the expected trajectory while the external force and torque on the bucket from the soil are applied. Operation space control is used for the manipulator. The whole simulation is shown in Fig. 11, which records the history trajectories of all the bodies of the sampling mechanism, and Fig. 12 indicates tracking performance for the expected trajectory of the tip. This simulation proves that the bucket can move along the expected circle, and the actual trajectory can track the desired trajectory accurately with only minimal error in the initial stage. Operation space control can make the bucket move along the expected trajectory even when the disturbance of the external variable load from lunar soil is applied.

The sampling mechanism status is shown in Fig. 13. The joint angle changes smoothly, and the position and attitude of the bucket can be tracked accurately. Though the displacement and the angular velocity have a little error at the beginning stage due to the external uncertain load, they converge to the expected trajectory soon later. Figure 13(f) indicates that the manipulator is mainly driven by joint 1 and joint 2 in the sampling process. The torque of joint 1 is about $35 \mathrm{Nm}$, and the torque of joint 2 vibrates around 0 with the amplitude less than $10 \mathrm{Nm}$. The torque of joint 3 is less than $5 \mathrm{Nm}$. The joint torque has some oscillations in the whole sampling process to adapt the external variable load applied to the bucket.

\section{Conclusions}

In this paper, the sampling process for lunar regolith was studied and the following conclusions were obtained:

(1) A modified 3D discrete element model for lunar regolith was built by considering the torque, bending and equivalent attraction between two particles. Microscopic parameters were calibrated based on a tri-axial simulation test, and coincided with the results of physical experiments from other literature.

(2) The dynamics model of the sampling manipulator was established using Lagrange formulations, and an operation space control schema was designed for the sampling procedure and validated using numerical simulation.

(3) The modeling and calibration methods for the lunar soil sampling in this investigation would support the performance analysis and design of the sampling mechanism in the future lunar explorations.

\section{Acknowledgments}

The corresponding author is Cheng Wei, his E-mail is: weicheng@ hit.edu.cn. This work was supported by the National Natural Science Foundation of China (51105100), Doctoral Fund of the Min- istry of Education of China (20112302120007), the China Postdoctoral Science Foundation (20110491050) and funds from the National Key Laboratory (HIT.KLOF.2011.075).

\section{References}

1) Mueller, R. P. and Van Susante, P. J.: A Review of Lunar Regolith Excavation Robotic Device Prototypes, AIAA SPACE 2011 Conference Exposition Online Proceedings, 2011, pp. 1719-1733.

2) Kubota, T., Kunii, Y. and Kuroda, Y.: Japanese Lunar Robotics Exploration by Co-Operation with Lander and Rover, J. Earth Syst. Sci., 114, 6 (2005), pp. 777-785.

3) Mitchell, J. K., Houston, W. N., Scott, R. F., Costes, N. C., Carrier, W. D., III and Bromwell, L. G.: Mechanical Properties of Lunar Soil: Density, Porosity, Cohesion and Angle of Internal Friction, Proceedings of the Third Lunar Science Conference (Supplement 3, Geochimica et Cosmochimica Acta), 1972, pp. 3235-3253.

4) Cundall, P. A. and Strack, O. D. L.: A Discrete Numerical Model for Granular Assemblies, Géotechnique, 29, 1 (1979), pp. 47-65.

5) Obermayr, M., Dressler, K., Vrettos, C. and Eberhard, P.: A BondedParticle Model for Cemented Sand, Comput. Geotech., 49 (2013), pp. 299-313.

6) Oida, A. and Momozu, M.: Simulation of Soil Behavior and Reaction by Machine Part by Means of DEM, Agric. Eng. Int. CIGR J. Sci. Res. Dev., IV (2002).

7) Jiang, M. J., Yu, H. S. and Harris, D.: A Novel Discrete Model for Granular Material Incorporating Rolling Resistance, Comput. Geotech., 32, 5 (2005), pp. 340-357.

8) Jiang, M. J., Shen, Z. F. and Thornton, C.: Microscopic Contact Model of Lunar Regolith for High Efficiency Discrete Element Analyses, Comput. Geotech., 54 (2013), pp. 104-116.

9) Matsushima, T., Katagiri, J., Uesugi, K., Tsuchiyama, A. and Nakano, T.: $3 \mathrm{~d}$ Shape Characterization and Image-Based Dem Simulation of the Lunar Soil Simulant Fjs-1, Aerospace Eng., 22, 1 (2009), pp. 15-23.

10) Matsushima, T., Katagiri, J., Uesugi, K., Tsuchiyama, A. and Nakano, T.: Image-Based Modeling of Lunar Soil Simulant for 3-D Dem Simulations, Earth \& Space 2006: Engineering, Construction, and Operations in Challenging Environments, Proceedings of the Tenth Biennial ASCE Aerospace Division International Conference on Engineering, Construction, and Operations in Challenging Environments, 2006, pp. 1-8.

11) Plackett, C. W.: A Review of Force Prediction Methods for Off-Road Wheels, J. Agric. Eng. Res., 31, 1 (1985), pp. 1-29.

12) Michaud, S., Richter, L., Patel, N., Thuer, T., Huelsing, T., Joudrier, L., Siegwart, R. and Ellery, A.: Rcet: Rover Chassis Evaluation Tools, Proceedings of the 8th ESA Workshop on Advanced Space Technologies for Robotics and Automation 'ASTRA 2004' ESTEC, Noordwijk, The Netherlands, 2004.

13) Taylor, B. P.: Experimental Evaluation and Semi-Empirical Modeling of the Tractive Performance of Rigid and Flexible Wheels on Lunar Soil Simulant, Virginia Polytechnic Institute and State University, Blacksburg, VA, 2009.

14) Andrade, G., Amar, F. B., Bidaud, P. and Chatila, R.: Modeling Robot-Soil Interaction for Planetary Rover Motion Control, Proceedings of the 1998 IEEE/RSJ Int. Conference on Intelligent Robots and Systems, 1998, pp. 576-581.

15) Hong, W.: Modeling, Estimation, and Control of Robot-Soil Interactions, Massachusetts Institute of Technology, Boston, 2001.

16) Bui, H. H., Kobayashi, T., Fukagawa, R. and Wells, J. C.: Numerical and Experimental Studies of Gravity Effect on the Mechanism of Lunar Excavations, J. Terramech., 46, 3 (2009), pp. 115-124.

17) Bui, H. H., Kobayashi, T. and Fukagawa, R.: Simulation of Soil Excavations on the Lunar Surface, 10th European Conference of the International Society for Terrain-Vehicle Systems, Budapest, 2006.

18) Carrier, III W. D., Olhoeft, G. R. and Mendell, W.: Physical Properties of the Lunar Surface, Lunar Sourcebook: A User's Guide to the Moon, Cambridge University Press, London, 1991, pp. 475-567.

19) Scott, R. F.: 27th Rankine Lecture-Failure, Geotechnique, 37, 4 (1987), pp. 423-466.

20) Perko, H., Nelson, J. and Sadeh, W.: Surface Cleanliness Effect on Lunar Soil Shear Strength, J. Geotech. Geoenviron. Eng., 27, 4 (2001), pp. 371-383. 